\title{
Novel Therapies in Lung Cancer Management
}

\section{An Update on the Role of Topotecan}

\author{
Guest Editor: Joan H. Schiller \\ University of Wisconsin Hospital, Madison, Wisc., USA
}

Lung cancer is far and away the leading cause of cancer-related deaths in the United States. According to recent statistics, 169,500 new cases of lung cancer will be diagnosed in 2001, with an estimated 157,400 deaths [1]. The majority of lung cancer patients present with disseminated (stage III or IV) disease for which they receive etoposide- and/or platinum-based chemotherapy. Although etoposide- and platinum-based chemotherapy have been associated with high initial antitumor response rates and improved survival and quality-of-life endpoints, such benefits have been modest and short-lived. As a consequence, death from recurrent chemotherapy-resistant metastatic disease remains the inevitable outcome in greater than $90 \%$ of patients. The identification of novel agents with activity in first-line and recurrent disease has been an unfulfilled priority for the past 20 years.

Although progress in the treatment of lung cancer has been disappointing over the past 20 years, there is now reason for optimism. A number of new cytotoxic agents with novel, nonoverlapping mechanisms of action have been introduced into the clinical armamentarium, including the pyrimidine analogue, gemcitabine; the vinca alkaloid, vinorelbine; the taxanes, paclitaxel and docetaxel; and the topoisomerase I inhibitors, topotecan and irinotecan. The use of these novel agents, with differing cellular targets and resistance profiles relative to standard chemo-

Supported by GlaxoSmithKline, Brentford, Middlesex, UK.

\begin{tabular}{ll}
\hline KARGER & @ 2001 S. Karger AG, Basel \\
Fax $+41613061234-2414 / 01 / 0615-0001 \$ 17.50 / 0$ \\
$\begin{array}{l}\text { E-Mail karger@karger.ch } \\
\text { www.karger.com }\end{array}$ & $\begin{array}{l}\text { Accessible online at: } \\
\text { www.karger.com/journals/ocl }\end{array}$
\end{tabular}

therapy, has the potential to slow or even prevent the development of resistant cells. Of these active new agents, topotecan (HYCAMTIN ${ }^{\circledR}$, GlaxoSmithKline, Brentford, Middlesex, UK) is a novel topoisomerase I inhibitor with potentially broad applicability in the treatment of solid and hematologic malignancies, including small-cell lung cancer (SCLC) and non-small-cell lung cancer (NSCLC).

This special issue of Oncology is devoted to updating the published clinical data related to the use of topotecan in SCLC and NSCLC. The articles presented herein were derived, in part, from presentations delivered at the Ninth World Congress of Lung Cancer in September 2000 in Tokyo, Japan. The World Congress of Lung Cancer, a triannual meeting convened by the world's leading authorities in the management of lung cancer, is an important venue for disseminating state-of-the-art information pertaining to the use of novel treatments in lung cancer therapy. The role of topotecan in the treatment of lung cancer was an important component of the conference and is the topic featured within this supplement.

Articles were contributed by leading experts in the management and treatment of lung cancer from the United States and abroad. This supplement is restricted to the review of cytotoxic agents; therefore, the new molecular-targeted drugs, such as vascular endothelial growth factor or epidermal growth factor inhibitors and antiangiogenesis drugs will not be discussed.

The supplement begins with an overview of current standards of care in SCLC and NSCLC (Joan H. Schiller).

\footnotetext{
Joan H. Schiller, MD

University of Wisconsin Hospital

Room K4/636, 600 Highland Avenue

Madison, WI 53792 (USA)

Tel. +1 608263 8600, Fax +1 608263 8613, E-Mail jhschill@facstaff.wisc.edu
} 
Platinum-based regimens (e.g., etoposide/cisplatin, etoposide/carboplatin) represent the mainstay treatment for SCLC and NSCLC. Although these regimens demonstrate significant antitumor activity and have extended the survival of patients relative to best supportive care, there remains a lack of long-term success as measured by the high proportion of patients who relapse and by the poor overall survival of both chemotherapy responders and nonresponders. The article concludes by underscoring the clinical need for new cytotoxic therapies with novel mechanisms of action. The next article by Chao Huang and Joseph Treat provides an overview of new agents being used in the treatment of lung cancer, with a focus on topoisomerase I inhibitors. A brief introduction into the use of topotecan, a novel topoisomerase I inhibitor, in second-line SCLC and its potential role in first- and secondline NSCLC and first-line SCLC is provided.

The focus subsequently shifts to a series of articles that overview the exciting new data for topotecan in first-line SCLC. In the phase I study by Penny O'Neill and Peter Clark et al., the feasibility and preliminary antitumor activity of intravenous topotecan administered with etoposide was established in previously untreated patients with extensive disease. The regimen was well tolerated, with high initial response rates reported. In the next SCLC article, John Eckardt reviews the feasibility of a more convenient oral formulation of topotecan in firstline SCLC. This phase II study was conducted in patients who were considered ineligible for standard etoposide- or platinum-based regimens. In this elderly patient population with multiple comorbidities, preliminary data demonstrate that oral topotecan was generally well tolerated and provided comparable antitumor activity relative to what has been reported for the standard intravenous regimen. These data, along with others, provide the rationale for further phase II/III investigation of oral topotecan in combination regimens in the first-line SCLC setting.

The subsequent series of articles summarizes the emerging data from studies investigating the potential role of topotecan in first-line NSCLC patients. In the initial NSCLC article by Roger Stupp et al., a well-tolerated regimen of intravenous topotecan with vinorelbine was established in advanced NSCLC patients. The topotecan/ vinorelbine combination exhibited a favorable toxicity profile relative to that associated with platinum-based regimens, with antitumor responses observed at all topotecan dose levels tested. In a comparable feasibility/tolerability study, J. Pujol reviews the tolerability of a regimen containing intravenous topotecan with carboplatin in advanced NSCLC. Preliminary data from this study suggested that topotecan with carboplatin is better tolerated relative to topotecan with cisplatin, with an improved nonhematologic toxicity profile. In the final NSCLC study, John Eckardt reports on the feasibility and preliminary antitumor activity of oral topotecan administered with intravenous paclitaxel in advanced NSCLC patients. The maximum tolerated dose of topotecan without granulocyte colony-stimulating factor support was established against a fixed dose of paclitaxel. This convenient regimen was well tolerated and demonstrated promising antitumor activity.

Finally, the future role of topotecan in the treatment of lung cancer and other solid and hematologic malignancies is reviewed (Joan H. Schiller). The route of administration (oral vs. intravenous), schedule (5-day, 3-day, weekly, continuous intravenous), and potential combination with other active agents represent important avenues for further investigation in phase II and III studies.

In summary, it is my hope that you will find this special issue of Oncology to be an important educational resource and to provide a state-of-the-art synopsis regarding the current role and future potential of topotecan in the treatment of SCLC and NSCLC. The preliminary data presented in this symposium provide sound rationale for continuing to pursue expanded use for this novel agent for the treatment of SCLC, NSCLC, and beyond.

\section{Reference}

1 Greenlee RT, Hill-Harmon MB, Murray T, Thun M: Cancer statistics, 2001. CA Cancer J Clin 2001;51:15-36. 